\title{
Nymphalidae, Papilionidae e Pieridae (Lepidoptera: Papilionoidea) da Serra do Sudeste do Rio Grande do Sul, Brasil
}

\author{
Ana Luiza Gomes Paz ${ }^{1,3}$; Helena Piccoli Romanowski'; Ana Beatriz Barros de Morais ${ }^{2}$ \\ ${ }^{1}$ Programa de Pós-Graduação em Biologia Animal, Departamento de Zoologia, Instituto de Biociências, \\ Universidade Federal do Rio Grande do Sul - UFRGS, Av. Bento Gonçalves, 9500, \\ Bloco IV, Prédio 43435, Sala 218, CEP 91501-970, Porto Alegre, RS, Brasil \\ ${ }^{2}$ Departamento de Biologia, Centro de Ciências Naturais e Exatas, \\ Universidade Federal de Santa Maria - UFSM, Faixa de Camobi, Km 09, \\ CEP 97105-900, Santa Maria, RS, Brasil \\ ${ }^{3}$ Autor para correspondência: Ana Luiza Gomes Paz,e-mail: algpaz@gmail.com
}

Paz, A. L. G.; Romanowski, H. P.; Morais, A. B. B. Nymphalidae, Papilionidae and Pieridae (Lepidoptera: Papilionoidea) of the Serra do Sudeste, Rio Grande do Sul, Brazil. Biota Neotrop., vol. 8, no. 1, Jan./Mar. 2008. Available from: <http://www.biotaneotropica.org.br/v8n1/en/abstract?inventory+bn01608012008>.

\begin{abstract}
To contribute to the knowledge on the composition and distribution of the butterfly fauna of the Southeastern region of Rio Grande do Sul state (RS), five areas at Serra do Sudeste were sampled between April/2003 and January/2004. The species composition of Nymphalidae, Papilionidae and Pieridae was compared with existing records for the contiguous areas at the foot of Serra do Sudeste and the South Coast Plain (Pelotas and surroundings). In a total of 289 net-hours of sampling at Serra do Sudeste, 2.326 individuals in 81 species were recorded: 59 Nymphalidae, 12 Papilionidae and 10 Pieridae. Literature data available for the adjacent areas lists 138 species. Putting both sources together, a total of 152 species are now registered for the Southeast Region of RS: 110 Nymphalidae, 14 Papilionidae and 28 Pieridae. Amongst these species, 14 (9\%) occur only in Serra do Sudeste, and 71 (47\%) only at the contiguous areas. The results demonstrate the peculiar composition of Serra do Sudeste lepidopterofauna and emphasize the biological importance of this area of Rio Grande do Sul.
\end{abstract}

Keywords: butterflies, species richness, conservation.

Paz, A. L. G.; Romanowski, H. P.; Morais, A. B. B. Nymphalidae, Papilionidae e Pieridae (Lepidoptera: Papilionoidea) da Serra do Sudeste do Rio Grande do Sul, Brasil. Biota Neotrop. vol. 8, no. 1, jan./mar. 2008. Disponível em: <http://www.biotaneotropica.org.br/v8n1/pt/abstract?inventory+bn01608012008>.

Resumo: Visando contribuir para conhecimento sistematizado da composição e distribuição da fauna de borboletas da região Sudeste do Rio Grande do Sul, entre abril de 2003 e janeiro de 2004, foram realizadas expedições para cinco locais na Serra do Sudeste. A composição de espécies de Nymphalidae, Papilionidae e Pieridae foi comparada aos registros existentes para as áreas adjacentes, na Encosta Sudeste e Litoral Sul, em Pelotas e seus arredores. No total de 289 horas-rede de amostragem, foram registrados 2.326 indivíduos e 81 espécies: 59 Nymphalidae, 12 Papilionidae e 10 Pieridae. Para a região adjacente, os trabalhos anteriores listam 138 espécies. Compilandose ambas as fontes, obtém-se para a região Sudeste do Estado, 152 espécies: 110 Nymphalidae, 14 Papilionidae e 28 Pieridae. Do total de espécies, 14 (9\%) ocorreram apenas na Serra do Sudeste e 71 (47\%) em Pelotas e seus arredores. Os resultados apresentam a composição peculiar da lepidopterfauna da Serra Sudeste, e corroboram a importância biológica desta área do Estado.

Palavras-chave: borboletas, riqueza de espécies, conservação. 


\section{Introdução}

As borboletas constituem cerca de $13 \%$ da ordem Lepidoptera e compreendem duas superfamílias (Papilionoidea e Hesperioidea). Distinguem-se da mariposas, sobretudo por apresentar antenas claviformes e hábitos predominantemente diurnos (Heppner 1991). No Brasil, ocorrem cerca de 3.280 espécies de borboletas (Brown Jr. \& Freitas 1999). Dentre as famílias de borboletas Nymphalidae, Papilionidae e Pieridae destacam-se por serem facilmente amostradas e reconhecíveis em campo, apresentarem populações residentes (Brown Jr. \& Freitas 2000b) e terem biologia relativamente bem conhecida, incluindo associação com planta hospedeira e hábitat (Sparrow et al. 1994).

No Rio Grande do Sul, o conhecimento da ocorrência e distribuição das espécies animais é escasso para muitos grupos, entre estes, borboletas. Listas regionais de espécies do grupo são importantes por prover informações sobre diversidade taxonômica, genética e ecológica (Motta 2002): também, através da comparação da ocorrência de espécies em diferentes habitats ou localidades podem ser definidas as formas de manejo mais adequadas (Balmer 2002). Assim, tais listas constituem material fundamental para avaliação e monitoramento ambiental e podem servir para determinar áreas prioritárias para conservação (De Vries et al. 1997, Harding et al. 1995, Viejo et al. 1989).

As florestas e campos nativos do Rio Grande do Sul têm sofrido intensa interferência humana. Registros históricos da vegetação original e fragmentos florestais atualmente maduros, secundários ou em recuperação são as únicas evidências do potencial florístico em algumas áreas (Quadros \& Pillar 2002). Atualmente, muitas áreas tropicais e subtropicais têm apenas fragmentos de florestas como resultado do desmatamento e, freqüientemente, estes contêm espécies que estão ameaçadas de extinção (Rogo \& Odulaja 2001). A fragmentação reduz o hábitat e a área de vida para plantas e animais. A destruição direta dos habitats, que vem ocorrendo em todas as partes do mundo, é a ameaça mais freqüente a borboletas e outros insetos. Assim, a manutenção de locais que sirvam como refúgios, tais como florestas ou campos nativos, é fundamental para sua conservação (New et al. 1995).

O Bioma Pampa, no qual se insere área de estudo, é o único bioma brasileiro que ocorre em apenas um Estado e está muito sujeito às regras de uso de terras de um único tipo, e bem aquém do desejável em termos de conservação se comparado ao restante do país. Por estes motivos, este bioma é muito ameaçado e qualquer alteração ambiental maior pode ser decisiva para seu futuro. Apesar disso, sua proteção tem sido negligenciada (Pillar et al 2006). Na parte sudeste deste bioma, localiza-se a Serra do Sudeste do Rio Grande do Sul, caracterizada pela topografia acidentada, grandes afloramentos rochosos e solos rasos (Boldrini 1997), com fisionomia peculiar, apresentando mistos de campos e matas; estas geralmente associadas a cursos d'água.

A Serra do Sudeste do Rio Grande do Sul é classificada como Área de Extrema Importância Biológica (Ministério do Meio Ambiente 2000). No entanto, nenhuma Unidade de Conservação foi de fato implementada nesta região. São raras as informações sobre os aspectos biológicos desta área do Estado. Com relação à fauna, as referências são ainda mais limitadas. Braun \& Braun (1980) e Belton (1994) elaboraram listagens de espécies de anfíbios e aves do Rio Grande do Sul, respectivamente, citando as espécies ocorrentes na Serra do Sudeste. Konrad \& Naeher (1996) apresentaram dados sobre as espécies de peixes, aves e mamíferos da Bacia do Rio Camaquã. Konrad \& Paloski (2000) analisaram a distribuição da ictiofauna da sub-bacia do Arroio João Dias (Minas do Camaquã, Caçapava do Sul, RS) e fornecem algumas informações gerais sobre a diversidade e distribuição de anfíbios, répteis, aves, mamíferos e invertebrados aquáticos comumente observados na região desta sub-bacia.

De uma maneira geral, até a última década, a maioria dos trabalhos existentes sobre a lepidopterofauna no Rio Grande do Sul havia sido desenvolvida com metodologias não padronizadas ou não explicitadas e objetivos diversos (Romanowski \& Buss 1997), o que dificulta comparações e análises ecológicas. Além disto, os locais de estudo não costumam ser identificados e, para muitas áreas do Estado, a ausência de dados é total. Quanto à Serra do Sudeste, em particular, não há dados sobre borboletas.

Biezanko \& Freitas (1938) estudaram lepidópteros em áreas adjacentes à Serra do Sudeste, que abrangem os atuais municípos de Pelotas, Capão do Leão e Morro Redondo. Segundo Krüger \& Silva (2003), as áreas por eles estudadas correspondem às regiões fisiográficas "Litoral Sul" e "Encosta" (esta, margeando a Serra do Sudeste) e assim será referida no presente estudo. No catálogo produzido para os insetos daquelas regiões, Litoral Sul e Encosta, Biezanko \& Freitas relataram a ocorrência de 51 espécies de Nymphalidae, 14 Pieridae e seis Papilionidae. Também para o Litoral Sul e a Encosta, Biezanko (1949, 1958, 1959, 1960a, b) gerou listas com registros de 98 espécies de Nymphalidae, 28 de Pieridae e 13 de Papilionidae.

Mais recentemente, Krüger \& Silva (2003) revisaram as espécies testemunho coletadas por Biezanko e Freitas (1938) e Biezanko (1949, 1958, 1959, 1960a, b) e atualizaram a nomenclatura. Mudanças na sistemática do grupo ocasionaram alterações no número de espécies originalmente citado. A partir desta revisão, as listas anteriormente produzidas para o Litoral Sul e a Encosta, resultam num total de 140 espécies: 104 Nymphalidae, 26 Pieridae e 10 Papilionidae. Krüger \& Silva (2003) também amostraram borboletas nas áreas que Biezanko havia amostrado. Encontraram um total de 89 espécies: 70 Nymphalidae, 11 Pieridae e oito Papilionidae. Destas, três foram novos registros para área, todos pertencentes à família Nymphalidae: Memphis moruus stheno (Prittwitz, 1865), Blepolenis batea batea (Hübner, 1822) e Splendeuptychia libitina (Butler, 1870).

A Serra do Sudeste localiza-se contiguamente à área estudada pelos autores acima citados. Pode-se supor certa similaridade na lepidopterofauna uma vez que as latitudes são semelhantes. Entretanto, por ser diferenciada fisionômica e florísticamente, sugere-se uma fauna distinta das demais áreas do Estado. O presente trabalho visa contribuir para o conhecimento da lepidopterofauna do Rio Grande do Sul, abordando a lacuna no conhecimento da fauna e, em particular, das borboletas ocorrentes na Serra do Sudeste.

\section{Material e Métodos}

Este trabalho foi efetuado, na região fisiográfica da Serra do Sudeste do Rio Grande do Sul, no Bioma Pampa, nos municípios

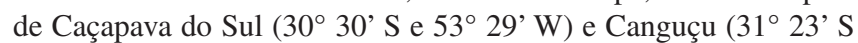
e $52^{\circ} 40^{\prime} \mathrm{W}$ ), que distam $140 \mathrm{~km}$ entre si e, respectivamente, $260 \mathrm{e}$ $300 \mathrm{~km}$ de Porto Alegre e 235 e $70 \mathrm{~km}$ de Pelotas (Figura 1).

A região tem relevo de ondulado a fortemente ondulado, com altitudes que variam de 150 a $500 \mathrm{~m}$. Geologicamente é a região mais antiga do Estado, Escudo Granítico, no Planalto Sul-Riograndense (Boldrini 1997). O clima da região corresponde ao Cfalg' de Köppen, clima úmido, sem estiagem, subtropical (ou "quase temperado"; Mota 1951). A precipitação média anual é de $1.350 \mathrm{~mm}$ e a temperatura média anual é de $17{ }^{\circ} \mathrm{C}$ (Girardi-Deiro et al. 2002). Porto (2002) classifica as formações vegetais como campestres e florestais de pequeno porte, os campos sendo do tipo sujo e vassourais, onde se estabelece uma tipologia de campo dotada de fisionomia grosseira, aproximando-se à savana.

Foram selecionadas cinco trilhas em áreas rurais que abrangessem as características fisionômicas da região e campos, bordas de mata e 


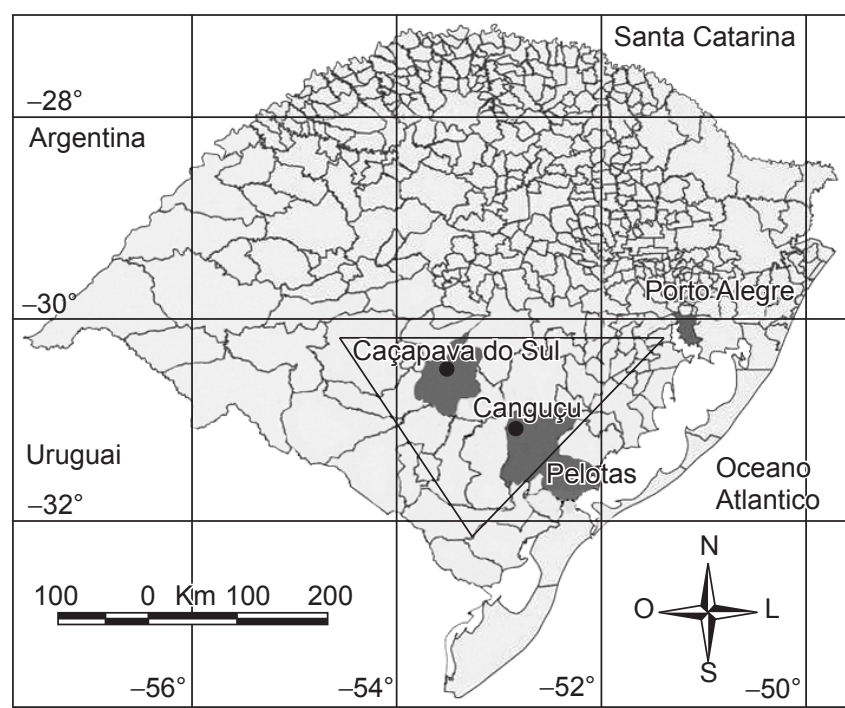

Figura 1. Limites dos municípios do Rio Grande do Sul e municípios (cinza escuro) das áreas de estudo (círculos pretos) na Serra do Sudeste do Rio Grande do Sul (triângulo). Mapa modificado a partir de Danielle Crawshaw (UFRGS, dados não publicados).

Figure 1. Study areas (black circles) and municipalities (dark grey) at Serra do Sudeste (triangle) in Rio Grande do Sul state. Black lines in the map indicate boundaries of Rio Grande do Sul municipalities. Map modified from Danielle Crawshaw (UFRGS, unpublished data).

matas, com relevo de moderadamente ondulado a acidentado e com solo sob níveis de antropização (sobretudo agropecuária) de mínimo a acentuado. Foram determinados dois locais de amostragem no interior do município de Caçapava do Sul e três no interior do município de Canguçu, respectivamente: Pedra do Segredo (30³2' S e 53 $33^{\prime} \mathrm{W}$; altitude: $195 \mathrm{~m}$ ): apresenta relevo muito abrupto, com sucessivas escarpas e grandes afloramentos rochosos em meio a áreas de campo ondulado. A trilha atravessa um mosaico de vegetação: pequenas porções de campo, borda e interior de mata de encosta, associada a um curso d'água. Devido à posição geográfica, a mata contém elementos da Floresta Atlântica, que não ocorrem nas demais áreas amostradas neste estudo. Atividades variadas de turismo e lazer são realizadas no local sem qualquer controle, deixando evidências de forte impacto derivado da visitação. Passo do Lajeado ( $30^{\circ} 40^{\prime} \mathrm{S}$ e $53^{\circ} 27^{\prime} \mathrm{W}$; altitude: $\left.160 \mathrm{~m}\right)$ : possui relevo de fracamente ondulado a plano. A trilha abrangeu um campo "sujo" utilizado para pecuária às margens de uma mata ciliar também com aspecto extremamente alterado, apresentando menos flores que nas demais trilhas. Rincão da Ronda - Campo ( $31^{\circ} 05^{\prime} \mathrm{S}$ e $52^{\circ}$ 52' W; altitude: $260 \mathrm{~m}$ ): área de campo com relevo fortemente ondulado, utilizada para pecuária extensiva. Flores principalmente de monocotiledôneas e de espécies características de sucessão secundária como algumas vassouras. Rincão da Ronda, Mata ( $31^{\circ} 06^{\prime} \mathrm{S}$ e $52^{\circ} 52^{\prime} \mathrm{W}$; altitude: $170 \mathrm{~m}$ ): vale em meio a relevo fortemente ondulado, adjacente a Rincão da Ronda, Campo. A trilha localizou-se no interior de mata ciliar, com pouca influência da pecuária extensiva. A mata tem altura em torno de 10 metros, é densa e apresenta algumas clareiras em seu interior. Coxilha do Fogo $\left(31^{\circ} 05^{\prime} \mathrm{S}\right.$ e 52 50' W; altitude $300 \mathrm{~m}$ ): mata úmida e sombreada, às margens de um pequeno curso d'água, em meio a campo ondulado. Possui flores abundantes, sobretudo na borda. A prática da pecuária extensiva é mais acentuada nesta trilha do que nas duas anteriormente citadas.

Foram realizadas cinco expedições entre abril de 2003 e janeiro de 2004. A amostragem baseou-se em metodologia adaptada a partir de Pollard (1977), seguindo Iserhard \& Romanowski (2004), Marchiori \& Romanowski (2006) e Dessuy e Morais (2007). Em cada uma das áreas foi determinado um transecto de cerca $1000 \mathrm{~m}$, que era percorrido por aproximadamente três horas em cada ocasião de amostragem, sempre no período entre 9 e 17 horas. A cada ocasião amostral, o turno (manhã ou tarde) em que cada transecto era amostrado era invertido, para evitar qualquer efeito horário-local. As amostragens foram realizadas apenas sob condições climáticas minimamente adequadas, isto é, sem chuva ou vento excessivo e com temperatura acima de $10-12^{\circ} \mathrm{C}$.

Em cada ocasião de amostragem, quatro amostradores experientes, em média, munidos de rede entomológica percorriam os transectos a passo lento, procurando ativamente por borboletas ao longo e ao redor da trilha, visando cobrir um espectro amplo de alturas de vôo. Borboletas avistadas eram capturadas com rede entomológica. Sempre que possível, a identificação era feita no local - se necessário, com o auxílio de guias de campo especializados (Canals 2000, 2003) - e, a seguir, o espécime liberado. Indivíduos distintamente conspícuos, passíveis de identificação segura sem a captura com rede foram amostrados apenas visualmente, visando manter a manipulação das borboletas ao mínimo necessário. Para cada indivíduo foram registrados em planilha de campo espécie, hora e local.

Tomou-se sempre o cuidado de amostrar a ampla gama de grupos de borboletas da assembléia estudada. Para tanto, eram inspecionados os mais variados substratos, desde o solo e vegetação rasteira, arbustos, até árvores altas e espaço aberto, seguindo recomendações em Brown (1972). Visou-se, assim, contemplar com a maior fidelidade possível a representatividade das formas menores, menos conspícuas ou raras. Quando aplicável, recorreu-se também ao uso de binóculos e fotografia digital como auxílio para identificação.

Quando a identificação segura de algum espécime não fosse possível em campo, este era coletado, acondicionado em envelope e levado a laboratório para montagem e identificação. Pelo menos um exemplar de cada espécie foi depositado como material-testemunho na coleção de referência do Laboratório de Ecologia de Insetos do Departamento de Zoologia da UFRGS. Entretanto, sempre absolutamente desnecessário, evitou-se o sacrifício de animais, visando uma metodologia o menos destrutiva possível (Freitas et al 2003).

Para a identificação, tomou-se como base a referida coleção, bibliografia especializada (Brown 1992, Canals 2000, 2003, D'Abrera 1981, 1984, 1987a, b, 1988, 1994, 1995, Tyler et al. 1994) e, conforme o caso, consulta a especialistas. O sistema de classificação adotado seguiu Brown Jr. (1992) e Freitas \& Brown Jr. (2004). A nomenclatura foi atualizada segundo Lamas (2004). Todos os dados gerados neste estudo foram adicionados ao banco de dados relacional BorbRS ${ }^{\circledR}$ e ao Banco de Imagens do Laboratório de Ecologia de Insetos do Departamento de Zoologia da UFRGS.

Em todo e qualquer caso em que restou qualquer dúvida quanto à identificação de algum indivíduo, este foi desconsiderado e não foi incluído nos resultados. As espécies, dentro das subfamílias, encontram-se listadas por ordem alfabética.

A lista de espécies de Nymphalidae, Papilionidae e Pieridae obtida na Serra do Sudeste foi comparada aos registros existentes para as áreas contíguas, Encosta Sudeste e Litoral Sul do Rio Grande do Sul, doravante referidos por B: registros de Biezanko e cols (Biezanko \& Freitas, 1938, Biezanko, 1949, 1958, 1959a, 1960a, b), atualizados de acordo com a revisão de Krüger \& Silva (2003); K\&S: registros de Krüger \& Silva (2003) nas mesmas áreas.

\section{Resultados e Discussão}

No total, 289 horas-rede de amostragem foram realizadas e registrados 2.326 indivíduos, pertencentes a 81 espécies de borboletas na Serra do Sudeste (Tabela 1).

Somando-se os dados revisados de $\mathbf{B}$ e K\&S, obtêm-se 138 espécies e subespécies de borboletas das famílias Nymphalidae, 
Paz, A. L. G. et al.

Tabela 1. Espécies de Nymphalidae, Papilionidae e Pieridae (Lepidoptera: Papilionoidea) do Sudeste do Rio Grande do Sul: SS - registros para a Serra do Sudeste (municípios de Caçapava do Sul e Canguçu), obtidos entre abril de 2003 e janeiro de 2004, B - registros para o município de Pelotas e seus arredores entre 1938 e 1960 (Biezanko \& Freitas, 1938; Biezanko, 1949, 1958, 1959, 1960a, 1960b), K\&S - registros para o município de Pelotas e seus arredores entre outubro de 1999 e junho de 2001 (Krüger \& Silva, 2003). (S) número de espécies; • espécies registradas na SS, não registradas nos trabalhos de B e K\&S; * espécies avistadas fora dos períodos regulares de amostragem. espécie pouco provável de ocorrer na área, necessitando confirmação.

Table 1. Species of Nymphalidae, Papilionidae e Pieridae (Lepidoptera: Papilionoidea) of the Southeastern region of Rio Grande do Sul state: SS - records for Serra do Sudeste (Caçapava do Sul and Canguçu municipalities), registered between April/2003 and January/2004, B - records for Pelotas and surroundings between 1938 and 1960 (Biezanko \& Freitas, 1938; Biezanko, 1949, 1958, 1959, 1960a, 1960b), K\&S - records for Pelotas and surroundings between October/1999 and Juny/2001 (Krüger \& Silva, 2003). (S) species richness; • species recorded at the SS, not recorded by B and K\&S; * species recorded out of the regular sampling periods. species of questionable occurrence in the area, needing confirmation.

\begin{tabular}{|c|c|c|c|}
\hline \multirow[t]{2}{*}{ Famílias/ Espécies } & \multirow[t]{2}{*}{ SS } & \multicolumn{2}{|c|}{$\mathbf{P A}$} \\
\hline & & B & K\&S \\
\hline \multicolumn{4}{|l|}{ NYMPHALIDAE $(\mathrm{S}=110)$} \\
\hline \multicolumn{4}{|l|}{ libytheinae $(S=1)$} \\
\hline Libytheana carinenta (Cramer, 1777) & - & $\mathrm{x}$ & $\mathrm{x}$ \\
\hline \multicolumn{4}{|l|}{ Danainae $(S=3)$} \\
\hline * Danaus erippus (Cramer, 1775) & $\mathrm{x}$ & $\mathrm{x}$ & $\mathrm{x}$ \\
\hline * Danaus gilippu gilippus (Cramer, 1775) & $\mathrm{x}$ & $\mathrm{x}$ & - \\
\hline Lycorea ilione (Cramer, 1775) & - & $\mathrm{x}$ & - \\
\hline \multicolumn{4}{|l|}{ Ithomiinae $(S=10)$} \\
\hline Dircenna dero (Hübner, 1823) & $\mathrm{x}$ & $\mathrm{x}$ & - \\
\hline Episcada carcinia Schaus, 1902 & - & $\mathrm{x}$ & - \\
\hline Epityches eupompe (Geyer, 1832) & $\mathrm{x}$ & $\mathrm{x}$ & $\mathrm{x}$ \\
\hline - Hypoleria adasa (Hewitson, [1855]) & - & $\mathrm{x}$ & $\mathrm{x}$ \\
\hline Mechanitis lysimnia lysimnia (Fabricius, 1793) & $\mathrm{x}$ & $\mathrm{x}$ & - \\
\hline Methona themisto (Hübner, 1818) & - & $\mathrm{x}$ & - \\
\hline Placidina euryanassa (Felder \& Felder, 1860) & - & $\mathrm{x}$ & $\mathrm{x}$ \\
\hline Episcada hymenaea hymenaea (Prittwitz, 1865) & - & $\mathrm{x}$ & - \\
\hline - Pseudoscada erruca (Hewitson, 1855) & $\mathrm{x}$ & - & - \\
\hline Pteronymia sylvo (Geyer, 1832) & - & $\mathrm{x}$ & - \\
\hline \multicolumn{4}{|l|}{ Charaxinae $(S=3)$} \\
\hline - Archaeoprepona chalciope (Hübner, [1823]) & $\mathrm{x}$ & - & - \\
\hline Memphis moruus stheno (Prittwitz, 1865) & - & - & $\mathrm{x}$ \\
\hline Zaretis itys (Cramer, 1777) & $\mathrm{x}$ & $\mathrm{x}$ & $\mathrm{x}$ \\
\hline \multicolumn{4}{|l|}{ Apaturinae $(S=3)$} \\
\hline Doxocopa kallina (Staudinger, 1886) & $\mathrm{x}$ & $\mathrm{x}$ & $\mathrm{x}$ \\
\hline Doxocopa laurentia (Godart, [1824]) & $\mathrm{x}$ & $\mathrm{x}$ & $\mathrm{x}$ \\
\hline Doxocopa zunilda (Godart, [1824]) & - & $\mathrm{x}$ & - \\
\hline \multicolumn{4}{|l|}{ Morphinae $(\mathrm{S}=2)$} \\
\hline Morpho aega (Hübner, [1822]) & - & $\mathrm{x}$ & $\mathrm{x}$ \\
\hline Morpho episthophus catenaria (Perry, 1811) & $\mathrm{x}$ & $\mathrm{x}$ & $\mathrm{x}$ \\
\hline \multicolumn{4}{|l|}{ Brassolinae $(\mathrm{S}=11)$} \\
\hline Blepolenis batea batea (Hübner, [1821]) & - & - & $\mathrm{x}$ \\
\hline - Blepolenis catharinae (Stichel, 1902) & $\mathrm{x}$ & - & - \\
\hline Brassolis astyra astyra Godart, [1824] & - & $\mathrm{x}$ & $\mathrm{x}$ \\
\hline Caligo illioneus (Cramer, 1775) & - & $\mathrm{x}$ & - \\
\hline Caligo martia (Godart, [1824]) & - & $\mathrm{x}$ & - \\
\hline Catoblepia amphirhoe (Hübner, [1825]) & - & $\mathrm{x}$ & - \\
\hline Dynastor darius darius (Fabricius, 1775) & - & $\mathrm{x}$ & - \\
\hline Eryphanis reevesii (Doubleday, [1849]) & $\mathrm{x}$ & $\mathrm{x}$ & $\mathrm{x}$ \\
\hline Opoptera sulcius (Staudinger, 1887) & - & $\mathrm{x}$ & - \\
\hline Opsiphanes invirae amplificatus Stichel, 1904 & - & $\mathrm{x}$ & $\mathrm{x}$ \\
\hline - Penetes pamphanis Doubleday, [1849] & $\mathrm{x}$ & - & - \\
\hline \multicolumn{4}{|l|}{ Satyrinae $(S=19)$} \\
\hline Capronnieria galesus (Godart, [1824]) & - & $\mathrm{x}$ & $\mathrm{x}$ \\
\hline
\end{tabular}


Tabela 1. Continuação...

\begin{tabular}{|c|c|c|c|}
\hline \multirow[t]{2}{*}{ Famílias/ Espécies } & \multirow[t]{2}{*}{ SS } & \multicolumn{2}{|c|}{$\mathbf{P A}$} \\
\hline & & B & K\&S \\
\hline Eteona tisiphone (Boisduval, 1836) & $\mathrm{x}$ & $\mathrm{x}$ & $\mathrm{x}$ \\
\hline Forsterinaria necys (Godart, [1824]) & $\mathrm{x}$ & $\mathrm{x}$ & $\mathrm{x}$ \\
\hline Forsterinaria quantius (Godart, [1824]) & - & $\mathrm{x}$ & $\mathrm{x}$ \\
\hline Godartiana muscosa (Butler, 1870) & - & $\mathrm{x}$ & - \\
\hline Hermeuptychia hermes (Fabricius, 1775) & $\mathrm{x}$ & $\mathrm{x}$ & $\mathrm{x}$ \\
\hline Moneuptychia paeon (Godart, [1824]) & - & $\mathrm{x}$ & $\mathrm{x}$ \\
\hline Moneuptychia soter (Butler, 1877) & $\mathrm{x}$ & $\mathrm{x}$ & $\mathrm{x}$ \\
\hline Pampasatyrus peryphas (Godart, [1824]) & $\mathrm{x}$ & $\mathrm{x}$ & - \\
\hline Pampasatyrus quies (Berg, 1877) & - & $\mathrm{x}$ & - \\
\hline Paryphthimoides eous (Butler, 1867) & - & $\mathrm{x}$ & $\mathrm{x}$ \\
\hline - Paryphthimoides phronius (Godart, [1824]) & $\mathrm{x}$ & - & - \\
\hline Paryphthimoides poltys (Prittwitz, 1865) & $\mathrm{x}$ & $\mathrm{x}$ & $\mathrm{x}$ \\
\hline Praepedaliodes phanias (Hewitson, 1862) & $\mathrm{x}$ & $\mathrm{x}$ & $\mathrm{x}$ \\
\hline Splendeuptychia libitina (Butler, 1870) & - & - & $\mathrm{x}$ \\
\hline Taygetis ypthima Hübner, [1821] & $\mathrm{x}$ & $\mathrm{x}$ & $\mathrm{x}$ \\
\hline Yphthimoides celmis (Godart, [1824]) & $\mathrm{x}$ & $\mathrm{x}$ & $\mathrm{x}$ \\
\hline Yphthimoides straminea (Butler, 1867) & - & $\mathrm{x}$ & - \\
\hline Zischkaia pacarus (Godart, [1824]) & - & $\mathrm{x}$ & $\mathrm{x}$ \\
\hline \multicolumn{4}{|l|}{ Limenitidinae $(S=8)$} \\
\hline Adelpha abia (Hewitson, 1850) & - & $\mathrm{x}$ & - \\
\hline Adelpha epizygis Fruhstorfer, 1915 & - & $\mathrm{x}$ & - \\
\hline Adelpha hyas (Doyère, [1840]) & - & $\mathrm{x}$ & - \\
\hline Adelpha mythra (Godart, [1824]) & $\mathrm{x}$ & $\mathrm{x}$ & $\mathrm{x}$ \\
\hline Adelpha poltius A. Hall, 1938 & - & $\mathrm{x}$ & $\mathrm{x}$ \\
\hline Adelpha thessalia indefecta Fruhstorfer, 1913 & $\mathrm{x}$ & $\mathrm{x}$ & - \\
\hline Adelpha syma (Godart, [1824]) & - & $\mathrm{x}$ & $\mathrm{x}$ \\
\hline Adelpha zea (Hewitson, 1850) & $\mathrm{x}$ & $\mathrm{x}$ & - \\
\hline \multicolumn{4}{|l|}{ Biblidinae $(\mathrm{S}=13)$} \\
\hline Biblis hyperia (Cramer, 1779) & $\mathrm{x}$ & $\mathrm{x}$ & - \\
\hline - Callicore pygas eucale (Fruhstorfer, 1916) & $\mathrm{x}$ & - & - \\
\hline Diaethria candrena (Godart, [1824]) & $\mathrm{x}$ & $\mathrm{x}$ & $\mathrm{x}$ \\
\hline Diaethria clymena meridionalis (H.W. Bates, 1864) & $\mathrm{x}$ & $\mathrm{x}$ & - \\
\hline Dynamine myrrhina (Doubleday, 1849) & $\mathrm{x}$ & $\mathrm{x}$ & $\mathrm{x}$ \\
\hline Epiphile hubneri Hewitson, 1861 & $\mathrm{x}$ & $\mathrm{x}$ & $\mathrm{x}$ \\
\hline Eunica eburnea Fruhstorfer, 1907 & $\mathrm{x}$ & $\mathrm{x}$ & $\mathrm{x}$ \\
\hline Eunica maja maja (Fabricius, 1775) & - & $\mathrm{x}$ & - \\
\hline Haematera pyrame (Hübner, [1819]) & $\mathrm{x}$ & $\mathrm{x}$ & $\mathrm{x}$ \\
\hline Hamadryas epinome (C. Felder \& R. Felder, 1867) & $\mathrm{x}$ & $\mathrm{x}$ & $\mathrm{x}$ \\
\hline Hamadryas februa februa (Hübner, [1823]) & $\mathrm{x}$ & $\mathrm{x}$ & - \\
\hline Marpesia petreus (Cramer, 1776) & $\mathrm{x}$ & $\mathrm{x}$ & - \\
\hline Temenis laothoe meridionalis Ebert, 1965 & - & $\mathrm{x}$ & - \\
\hline \multicolumn{4}{|l|}{ Nymphalinae $(S=17)$} \\
\hline Anartia amathea roeselia (Eschscholtz, 1821) & $\mathrm{x}$ & $\mathrm{x}$ & $\mathrm{x}$ \\
\hline Anartia jatrophae jatrophae (Linnaeus, 1763) & - & $\mathrm{x}$ & - \\
\hline Colobura dirce (Linnaeus, 1758) & - & $\mathrm{x}$ & - \\
\hline Eresia lansdorfi (Godart, 1819) & - & $\mathrm{x}$ & - \\
\hline Hypanartia bella (Fabricius, 1793) & $\mathrm{x}$ & $\mathrm{x}$ & $\mathrm{x}$ \\
\hline Hypanartia lethe (Fabricius, 1793) & $\mathrm{x}$ & $\mathrm{x}$ & $\mathrm{x}$ \\
\hline Junonia evarete (Cramer, 1779) & $\mathrm{x}$ & $\mathrm{x}$ & $\mathrm{x}$ \\
\hline Ortilia ithra (W.F. Kirby, 1900) & $\mathrm{x}$ & $\mathrm{x}$ & $\mathrm{x}$ \\
\hline
\end{tabular}


Tabela 1. Continuação...

\begin{tabular}{|c|c|c|c|}
\hline \multirow[t]{2}{*}{ Famílias/ Espécies } & \multirow[t]{2}{*}{ SS } & \multicolumn{2}{|c|}{ PA } \\
\hline & & $\mathbf{B}$ & K\&S \\
\hline Ortilia liriope (Cramer, 1775) & - & $\mathrm{x}$ & $\mathrm{x}$ \\
\hline Ortilia orthia (Hewitson, 1864) & $\mathrm{x}$ & $\mathrm{x}$ & $\mathrm{x}$ \\
\hline Siproeta epaphus trayja (Hubner, [1823]) & $\mathrm{x}$ & $\mathrm{x}$ & $\mathrm{x}$ \\
\hline Siproeta stelenes meridionalis (Fruhstorfer, 1909) & $\mathrm{x}$ & $\mathrm{x}$ & $\mathrm{x}$ \\
\hline Tegosa claudina (Eschscholtz, 1821) & $\mathrm{x}$ & $\mathrm{x}$ & $\mathrm{x}$ \\
\hline Tegosa orobia (Hewitson, 1824) & $\mathrm{x}$ & $\mathrm{x}$ & $\mathrm{x}$ \\
\hline Telenassa teletusa (Godart, [1824]) & - & $\mathrm{x}$ & $\mathrm{x}$ \\
\hline Vanessa braziliensis (Moore, 1883) & $\mathrm{x}$ & $\mathrm{x}$ & $\mathrm{x}$ \\
\hline Vanessa carye (Hübner, [1812]) & - & $\mathrm{x}$ & $\mathrm{x}$ \\
\hline \multicolumn{4}{|l|}{ Heliconinae $(S=20)$} \\
\hline Actinote carycina Jordan, 1913 & $\mathrm{x}$ & $\mathrm{x}$ & $\mathrm{x}$ \\
\hline Actinote catarina Penz, 1996 & - & $\mathrm{x}$ & - \\
\hline Actinote discrepans D'Almeida, 1958 & - & $\mathrm{x}$ & $\mathrm{x}$ \\
\hline Actinote genitrix D’Almeida, 1922 & - & $\mathrm{x}$ & $\mathrm{x}$ \\
\hline Actinote mamita (Burmeister, 1861) & - & $\mathrm{x}$ & $\mathrm{x}$ \\
\hline - Actinote melanisans Oberthür, 1917 & $\mathrm{x}$ & - & - \\
\hline Actinote parapheles Jordan, 1913 & - & $\mathrm{x}$ & $\mathrm{x}$ \\
\hline Actinote pellenea Hübner, [1821] & - & $\mathrm{x}$ & $\mathrm{x}$ \\
\hline Actinote rhodope D'Almeida, 1923 & $\mathrm{x}$ & $\mathrm{x}$ & $\mathrm{x}$ \\
\hline Actinote surima (Schaus, 1902) & - & $\mathrm{x}$ & - \\
\hline Actinote thalia pyrrha (Fabricius, 1775) & $\mathrm{x}$ & $\mathrm{x}$ & $\mathrm{x}$ \\
\hline Agraulis vanillae maculosa (Stichel, [1908]) & $\mathrm{x}$ & $\mathrm{x}$ & $\mathrm{x}$ \\
\hline Dione juno juno (Cramer, 1779) & $\mathrm{x}$ & $\mathrm{x}$ & $\mathrm{x}$ \\
\hline Dione moneta moneta Hübner, [1825] & - & $\mathrm{x}$ & - \\
\hline Dryas iulia alcionea (Cramer, 1779) & $\mathrm{x}$ & $\mathrm{x}$ & $\mathrm{x}$ \\
\hline Eueides aliphera (Godart, 1819) & - & $\mathrm{x}$ & - \\
\hline Eueides isabella dianasa (Hübner, [1806]) & - & $\mathrm{x}$ & - \\
\hline Euptoieta hortensia (Blanchard, 1852) & $\mathrm{x}$ & $\mathrm{x}$ & $\mathrm{x}$ \\
\hline Heliconius erato phyllis (Fabricius, 1775) & $\mathrm{x}$ & $\mathrm{x}$ & $\mathrm{x}$ \\
\hline - Heliconius ethilla narcaea Godart, 1819 & $\mathrm{x}$ & - & - \\
\hline \multicolumn{4}{|l|}{$\begin{array}{l}\text { PAPILIONIDAE }(S=14) \\
\text { papilioninae }(S=14)\end{array}$} \\
\hline Battus polydamas polydamas (Linnaeus, 1758) & $\mathrm{x}$ & $\mathrm{x}$ & $\mathrm{x}$ \\
\hline - Battus polystictus polystictus (Butler, 1874) & $\mathrm{x}$ & - & - \\
\hline Heraclides anchisiades capys (Hubner, [1809]) & $\mathrm{x}$ & $\mathrm{x}$ & $\mathrm{x}$ \\
\hline Heraclides astyalus astyalus (Godart, 1819) & $\mathrm{x}$ & $\mathrm{x}$ & $\mathrm{x}$ \\
\hline Heraclides hectorides (Esper, 1794) & $\mathrm{x}$ & $\mathrm{x}$ & $\mathrm{x}$ \\
\hline Heraclides thoas brasiliensis (Rothschild \& Jordan, 1906) & $\mathrm{x}$ & $\mathrm{x}$ & $\mathrm{x}$ \\
\hline - Mimoides lysithous eupatorion (Lucas, [1859]) & $\mathrm{x}$ & - & - \\
\hline Mimoides lysithous rurik (Eschscholtz, 1821) & $\mathrm{x}$ & $\mathrm{x}$ & $\mathrm{x}$ \\
\hline - Parides agavus (Drury, 1782) & $\mathrm{x}$ & - & \\
\hline - Parides anchises nephalion (Godart, 1819) & $\mathrm{x}$ & - & - \\
\hline Parides bunichus perrhebus (Boisduval, 1836) & $\mathrm{x}$ & $\mathrm{x}$ & $\mathrm{x}$ \\
\hline Protesilaus helios (Rothschild \& Jordan, 1906) & $\mathrm{x}$ & $\mathrm{x}$ & - \\
\hline Pterourus hellanichus (Hewitson, 1868) & - & $\mathrm{x}$ & - \\
\hline Pterourus scamander (Boisduval, 1836) & - & $\mathrm{x}$ & $\mathrm{x}$ \\
\hline \multirow{2}{*}{\multicolumn{4}{|c|}{$\begin{array}{l}\text { PIERIDAE }(S=28) \\
\text { dismorphiinae }(S=5)\end{array}$}} \\
\hline & & & \\
\hline Dismorphia astyocha Hübner, [1831] & - & $\mathrm{x}$ & - \\
\hline Dismorphia thermesia (Godart, 1819) & - & $\mathrm{x}$ & - \\
\hline - Enantia lina psamathe (Fabricius, 1793) & $\mathrm{x}$ & - & - \\
\hline Enantia melite (Linnaeus, 1763) & - & $\mathrm{x}$ & - \\
\hline Pseudopieris nehemia (Boisduval, 1836) & $\mathrm{x}$ & $\mathrm{x}$ & - \\
\hline \multicolumn{4}{|l|}{ Pierinae $(S=11)$} \\
\hline Ascia monuste orseis (Godart, 1819) & - & $\mathrm{x}$ & $\mathrm{x}$ \\
\hline
\end{tabular}


Tabela 1. Continuação...

\begin{tabular}{|c|c|c|c|}
\hline \multirow[t]{2}{*}{ Famílias/ Espécies } & \multirow[t]{2}{*}{ SS } & \multicolumn{2}{|c|}{ PA } \\
\hline & & B & K\&S \\
\hline Catasticta bithys (Hübner, [1831]) & - & $\mathrm{x}$ & - \\
\hline Glutophrissa drusilla (Cramer, 1777) & - & $\mathrm{x}$ & - \\
\hline - Hesperocharis anguitia (Godart, 1819) & $\mathrm{x}$ & - & - \\
\hline Hesperocharis leucania (Boisduval, 1836) & - & $\mathrm{x}$ & $\mathrm{x}$ \\
\hline Leucidia brephos (Hübner, [1809]) & - & $\mathrm{x}$ & - \\
\hline Melete lycimnia petronia Fruhstorfer, 1907 & - & $\mathrm{x}$ & - \\
\hline Pereute antodyca (Boisduval, 1836) & - & $\mathrm{x}$ & $\mathrm{x}$ \\
\hline Pereute swainsoni (Gray, 1832) & - & $\mathrm{x}$ & - \\
\hline Tatochila autodice (Hübner, 1818) & - & $\mathrm{x}$ & $\mathrm{x}$ \\
\hline Theochila maenacte (Boisduval, 1836) & - & $\mathrm{x}$ & $\mathrm{x}$ \\
\hline \multicolumn{4}{|l|}{ Coliadinae $(\mathrm{S}=12)$} \\
\hline Aphrissa statira (Cramer, 1777) & - & $\mathrm{x}$ & - \\
\hline Colias lesbia lesbia (Fabricius, 1775) & $\mathrm{x}$ & $\mathrm{x}$ & $\mathrm{x}$ \\
\hline Eurema albula (Cramer, 1775) & $\mathrm{x}$ & $\mathrm{x}$ & $\mathrm{x}$ \\
\hline Eurema deva (Doubleday, 1847) & - & $\mathrm{x}$ & $\mathrm{x}$ \\
\hline Eurema elathea (Cramer, 1777) & $\mathrm{x}$ & $\mathrm{x}$ & - \\
\hline Eurema phiale (Cramer, 1775) & - & $\mathrm{x}$ & - \\
\hline Phoebis argante (Fabricius, 1775) & - & $\mathrm{x}$ & - \\
\hline Phoebis neocypris (Hübner, [1823]) & $\mathrm{x}$ & $\mathrm{x}$ & $\mathrm{x}$ \\
\hline Phoebis philea philea (Linnaeus, 1763) & $\mathrm{x}$ & $\mathrm{x}$ & $\mathrm{x}$ \\
\hline Phoebis sennae sennae (Linnaeus, 1758) & - & $\mathrm{x}$ & $\mathrm{x}$ \\
\hline Pyrisitia leuce (Boisduval, 1836) & $\mathrm{x}$ & $\mathrm{x}$ & - \\
\hline Rhabdodryas trite banksi (Breyer, 1939) & $\mathrm{x}$ & $\mathrm{x}$ & - \\
\hline Número total de espécies & 81 & 136 & 85 \\
\hline
\end{tabular}

Papilionidae e Pieridae, ocorrentes em Pelotas e arredores. Considerando estas e mais as espécies registradas no presente estudo totalizase 152 espécies de borboletas (110 Nymphalidae, 14 Papilionidae e 28 Pieridae) para a Região Sudeste do Estado, abrangendo Serra, Encosta e Litoral.

Para comparação entre dados de diferentes fontes, deve-se considerar que as condições de relevo, composição florística, topografia, micro-clima e os diferentes esforços amostrais aplicados nas investigações têm influência nos resultados observados. Quanto maior a extensão temporal dos inventários, maior tendência a aumentar o número total de espécies registradas (Summervile et al. 2001). Outro fator importante é a antropização, já mencionada por Biezanko (1960) e $\mathbf{K} \& \mathbf{S}$, e que se mostra cada vez mais acentuada, com expansão imobiliária, pecuária, agricultura e, crescentemente, com a silvicultura para extrativismo. As pesquisas de $\mathbf{B}$ datam de quatro a seis décadas atrás. Os locais, embora alguns desde então já fossem zonas urbanas ou urbanizadas, diante dos avanços das cidades, vem sofrendo interferência antrópica cada vez mais intensa ao longo deste período. Atualmente, várias das áreas por eles amostradas encontram-se dentro do perímetro urbano da cidade de Pelotas ou em áreas públicas. Além disso, seus estudos consistem de relatos da ocorrência de espécies a partir de observações dos autores, sem restrições quanto ao período amostral. Assim, poder-se-ia esperar o registro de um maior número de espécies em $\mathbf{B}$. Através dos resultados de $\mathbf{K} \boldsymbol{\&} \mathbf{S}$ para as mesmas áreas podemos apenas inferir os efeitos do impacto gerado por fatores antrópicos (em escala temporal), refletidos na lepidopterofauna.

A Serra do Sudeste localiza-se em região contígua, mas fisiograficamente diferente das anteriores, com composição florística particular. Apesar das diferenças entre as metodologias dos registros entre o presente estudo e o de $\mathbf{K} \boldsymbol{\&} \mathbf{S}$, tomando-o como base, uma vez que foi realizado numa escala de tempo razoavelmente comparável, permitimo-nos sugerir, ainda que com cautela, que há diferenças reais entre a lepidopterofauna das regiões de cada estudo como, por exemplo, espécies típicas da Serra e da Encosta/Litoral. Tal aspecto merece ser investigado em estudos futuros.

Com relação à composição de espécies, na Serra do Sudeste Nymphalidae apresentou maior representatividade (73\%), seguida de Papilionidae $(15 \%)$ e Pieridae $(12 \%)$. Esta composição diferiu daquela registrada na Encosta/Litoral, onde a família Pieridae (19\%) foi marcadamente mais representativa que Papilionidae (7\%). Biezanko $(1958,1959)$ salientou a alta riqueza de espécies de Pieridae nas áreas por ele amostradas, enquanto Papilionidae era pobremente representada na região em relação aos estados do centro e norte do país. Estas variações podem estar relacionadas à especificidade do hábitat e viabilidade de recursos para as espécies de borboletas. Pieridae são especialmente abundantes em áreas abertas e possuem muitos representantes comuns em áreas antrópicas (Brown Jr. \& Freitas 1999, Owen 1971). Lagartas de muitas espécies desta família se alimentam de leguminosas e algumas, de crucíferas cultivadas, chegando a tornarem-se pragas (Biezanko 1958).

Muitas espécies de Papilionidae podem ser indicadoras de matas bem conservadas e recursos hídricos abundantes, porém, algumas espécies são associadas a áreas abertas e até mesmo urbanas (Brown Jr. \& Freitas 1999). Grande parte dos papilionídeos da Serra do Sudeste foi registrada em borda de mata, clareiras ou beira de córregos. A maioria das espécies desta família ocorreu na trilha com a fisionomia mais heterogênea amostrada neste estudo.

Para a região Sudeste do Rio Grande do Sul, entre as subfamílias de Nymphalidae, Satyrinae, Heliconiinae, Nymphalinae e Biblidinae foram as mais ricas, entretanto as ordens de riqueza diferem entre 
os trabalhos. Esta riqueza total foi fortemente influenciada pelo elevado número de espécies destas subfamílias na Encosta/Litoral, sobretudo, devido aos dados de B. As subfamílias de Nymphalidae com maior número de espécies na Serra do Sudeste nesta investigação foram: Nymphalinae e Biblidinae $(S=11)$, seguidas por Satyrinae e Heliconiinae $(S=10)$.

Segundo Brown Jr. \& Freitas (2000a), espécies da subfamília Nymphalinae podem indicar perturbação (natural ou antrópica) e as da subfamília Satyrinae se relacionam negativamente com perturbação antrópica, sendo sensíveis a fragmentação, perda de habitat e poluição. Brassolinae, subfamília marcadamente rica na região da Encosta/Litoral, responde à variação de temperatura e mosaico de vegetação, refletindo suas plantas hospedeiras que ocorrem em manchas no sub-bosque (Brown Jr. \& Freitas 2000a, b).

Observando-se a composição total de espécies, 14 (9\%) espécies ocorreram apenas na Serra do Sudeste, não tendo sido registradas nas áreas contíguas. Em contraste, 71 (47\%) espécies foram registradas apenas na Encosta/Litoral. Destas últimas, 39 (25\%) foram registradas somente por $\mathbf{B}$ e três $(2 \%)$ somente por K\&S. Embora sem esforço amostral explicitado nas publicações, as pesquisas de B se estenderam por duas décadas, enquanto o presente estudo e o trabalho de $\mathbf{K} \boldsymbol{\&} \mathbf{S}$ foram executados em um período de no máximo 2 anos. Todavia, apesar das limitações impostas a comparações pelas diferentes intensidades amostrais empregadas em cada estudo, algumas considerações podem ser feitas.

Algumas das borboletas exclusivas, para ambas as áreas, são especialistas associadas a matas. Insetos especializados podem ser mais vulneráveis à extinção em habitats fragmentados do que os generalistas, possivelmente devido à sua necessidade de interação com recursos específicos em uma área maior (Shahabuddin \& Terborgh 1999), ressaltando a importância das florestas para conservação desta fauna.

Das espécies amostradas na Serra do Sudeste, 15 foram registradas anteriormente em $\mathbf{B}$, não tendo sido encontradas nos inventários de K\&S. Dentre estas, Pseudopieris nehemia (Boisduval, 1836) (Pieridae) foi classificada por Biezanko (1958) como escassa, encontrada em lugares úmidos. No presente estudo esta espécie foi registrada apenas no local menos antropizado, em interior da mata ciliar, onde sua planta hospedeira, Calliandra tweedii Benth. (Mimosaceae), era abundante.

Das quatro espécies pertencentes à família Papiliniodae ocorrentes apenas na Serra, três são associadas a florestas: Battus polystictus polystictus (Butler, 1874), Mimoides lysitous eupatorion (Lucas, 1859) e Parides agavus (Drury, 1782). A outra, Parides anchises nephalion (Godart, 1819) é mais resistente e ocorre em muitos hábitats, inclusive antrópicos (Brown Jr. 1992, Schwartz \& Di Mare 2001) e ocorreu nas áreas mais antropizadas.

Estes dados, mesmo tendo sido obtidos a partir de inventário de relativamente curto prazo, espelham a composição peculiar da lepidopterofauna da Serra Sudeste, e corroboram a importância biológica desta área do Estado. Como forma de preservar as características ambientais remanescentes da região é extremamente importante a consolidação de Unidades de Conservação abrangendo as fisionomias de campo e mata do norte e sul desta região fisiográfica. Estas áreas não diferem apenas na fisionomia entre os dois municípios: alto percentual de espécies registrado apenas em um destes $(27 \%$ das espécies de Canguçu e $28 \%$ das espécies de Caçapava do Sul) reflete a grande especificidade de suas faunas.

Nas áreas de relevo fortemente ondulado e matas ciliares características do sul da Serra do Sudeste há o pretendido Parque Estadual do Podocarpus. Todavia, embora constando na lista de Unidades de Conservação do Estado, abrange área mínima e nunca foi de fato implantado. No limite com a Depressão Central, ao norte, onde o relevo é mais abrupto o uso indiscriminado pelo turismo tem deixado marcas evidentes no ambiente. Apesar disso, há expressiva e peculiar taxocenose de borboletas, que merece ser conservada.

\section{Agradecimentos}

Aos colegas do Laboratório de Ecologia de Insetos (UFRGS) pela ajuda ao longo do trabalho. Ao Dr. Gilson R.P. Moreira (UFRGS) por disponibilizar sua propriedade, no município de Canguçu, para desenvolvimento deste estudo. Aos doutores Keith Brown Jr. e André Victor Lucci Freitas (UNICAMP) e Dra. Carla Penz (University of New Orleans) pelas identificações de borboletas. Ao CNPq pelas bolsas concedidas e pelo financiamento de parte do trabalho (Processo No478787 / 2001-4)

Contribuição $n^{\circ} 469$ do Departamento de Zoologia do Instituto de Biociências da Universidade Federal do Rio Grande do Sul.

\section{Referências Bibliográficas}

BALMER, O. 2002. Species lists in ecology and conservation: abundances matter. Conserv. Biol. 16(4):1160-1161.

BELTON, W. 1994. Aves do Rio Grande do Sul - Distribuição e biologia. Unisinos, São Leopoldo, 584p.

BIEZANKO, C.M. 1949. Acraeidae, Heliconidae e Nymphalidae de Pelotas e seus arredores. Arquivos de Entomologia. Série A, Edição do autor, Pelotas, p.1-16.

BIEZANKO, C.M. 1958. Pieridae da Zona Sueste do Rio Grande do Sul. Arquivos de Entomologia. Série A, Edição do autor, Pelotas, p.1-15.

BIEZANKO, C.M. 1959. Papilionidae da Zona Sueste do Rio Grande do Sul. Arquivos de Entomologia. Série A, Edição do autor, Pelotas,p.1-17.

BIEZANKO, C.M. 1960a. Danaidae et Ithomiidae da Zona Sueste do Rio Grande do Sul. Arquivos de Entomologia. Série A, Edição do autor, Pelotas, p.1-6.

BIEZANKO, C.M. 1960b. Satyridae, Morphidae et Brassolidae da Zona Sueste do Rio Grande do Sul. Arquivos de Entomologia. Série A, Edição do autor, Pelotas, p.1-13.

BIEZANKO, C.M. \& FREITAS, R.G. 1938. Catálogo dos insetos encontrados na cidade de Pelotas e seus arredores. Fasc. 1 - Lepidópteros. Boletim $\mathrm{N}^{\mathrm{o}} 25$ da Escola de Agronomia Eliseu Maciel, Edição do autor, Pelotas, p.1-32.

BOLDRINI, I.I. 1997. Campos do Rio Grande do Sul: Caracterização fisionômica e problemática ocupacional. Boletim do Instituto de Biociências, 56:1-39.

BRAUN, P. C. \& BRAUN, C. A. S. 1980. Lista prévia dos anfíbios do estado do Rio Grande do Sul, Brasil. Iheringia Zool. 56:121-146.

BROWN JR., K.S. 1972. Maximizing daily butterfly counts. Journal of Lepidopterist Society 26(3): 183-196.

BROWN JR., K.S. 1991. Conservation of Neotropical environments: Insects as indicators. In: The conservation of insects and their habitats (N. M. Collins \& J.A. Thomas, eds.). Academic Press. London, p.350-404.

BROWN JR., K.S. 1992. Borboletas da Serra do Japi: diversidade, hábitats, recursos alimentares e variação temporal. In História natural da Serra do Japi: Ecologia e preservação de uma área florestal no sudeste do Brasil (L.P.C. Morellato, org.). Campinas, Editora da Unicamp/Fapesp, p. $142-187$.

BROWN-Jr., K.S. \& FREITAS, A.V. L. 1999. Lepidoptera. In Joly, C. A. e C.E.M Bicudo (orgs). Biodiversidade do estado de São Paulo, Brasil: Síntese do conhecimentoao final do século xx, Volume 5 (C.R.F. Brandão \& E. M. Cancello, eds.), Invertebrados terrestres. Fapesp, São Paulo, p. 225-243.

BROWN JR., K. S. \& FREITAS, A. V. L. 2000a. Atlantic Forest Butterflies: indicators for landscape conservation. Biotropica, 32(4b):934-956.

BROWN JR., K. S. \& FREITAS, A. V. L. 2000b. Diversidade de Lepidoptera em Santa Tereza, Espírito Santo. Boletim do Museu de Biologia Mello Leitão, 11-12:71-118. 
CANALS, G.R. 2000. Mariposas Bonaerenses. Buenos Aires, L.O.L.A., $347 \mathrm{p}$.

CANALS, G.R. 2003. Mariposas de Misiones. Buenos Aires, L.O.L.A., 492p.

D'ABRERA, B. 1981. Butterflies of the Neotropical Region. Part I. Papilionidae \& Pieridae. Hill House, Victoria, XIV + 172p.

D’ABRERA, B. 1984. Butterflies of the Neotropical Region. Part II. Danaidae, Ithomidae, Heliconidae \& Morphidae. Hill House, Victoria, XIII + p. 174-384.

D'ABRERA, B. 1987a. Butterflies of the Neotropical Region. Part III. Brassolidae, Acraeidae \& Nymphalidae (partim). Hill House, Victoria, IX + p. 386-525.

D'ABRERA, B. 1987b. Butterflies of the Neotropical Region. Part IV. Nymphalidae (partim). Hill House, Victoria,XV + p. 528-678.

D'ABRERA, B. 1988. Butterflies of the Neotropical Region. Part V. Nymphalidae (conc.) \& Satyridae. Hill House, Victoria, IX + p. 680-877.

D'ABRERA, B. 1994. Butterflies of the Neotropical Region. Part VI. Riodinidae. Hill House, Victoria, IX + p. 880-1096.

D'ABRERA, B. 1995. Butterflies of the Neotropical Region. Part VII. Lycaenidae. Hill House, Victoria, XI + p. 1098-1270.

DESSUY, M. B. \& MORAIS, A.B.B. 2007. Diversidade de borboletas (Lepidoptera, Papilionoidea e Hesperioidea) em fragmentos de Floresta Estacional Decidual em Santa Maria, Rio Grande do Sul, Brasil. Revta. bras. Zool. 24(1):108-120.

DE-VRIES, P.J., MURRAY, D. \& LANDE, R. 1997. Species diversity in vertical, horizontal and temporal dimensions of a fruit-feeding butterfly community in an Equadorian rainforest. Biological Journal of the Linnean Society, 62:343-364.

FREITAS, A.V.L. \& K.S. BROWN JR. 2004. Phylogeny of the Nymphalidae (Lepidoptera). Systematic Biology, Washington, 53(3): 1-25.

FREITAS, A.V.L., FRANCINI, R.B \& BRONW JR, K.S. 2003. Insetos como indicadores ambientais. Capítulo 5 In Métodos de estudos em biologia da conservação e manejo da vida silvestre (L Cullen Jr, C. Valladares-Pádua \& R. Rudran, orgs.). Editora da UFPR, p. 125-151.

GIRARDI-DEIRO, A.M., OLIVEIRA, A. S. \& GOMES, K. E. 2002. Contribuição ao estudo das gramíneas e leguminosas da Serra do Sudeste, Rio Grande do Sul, Brasil. Revista Científica Rural, 7(1):34-41.

HARDING, P. T.; ASHER J. \& YATES, T. J. 1995. Butterfly Monitoring: 1 - Recording the changes. In Ecology and conservation of butterflies (A. S. Pullin, ed.). Chapman \& Hall, London, p.3-22.

HEPPNER, J.B. 1991. Faunal regions and the diversity of Lepidoptera. Tropical Lepidoptera, 2(1):1-85.

ISERHARD, C.A. \& ROMANOWSKI, H.P. 2004. Lista de espécies de borboletas (Lepidoptera, Papilionoidea e Hesperioidea) da região do vale do rio Maquiné, Rio Grande do Sul, Brasil. Revta. bras. Zool. 21(3): 649-662.

KONRAD, H.G. \& NAEHER N.I.P. 1996. Caracterização, diagnóstico e planejamento da bacia de drenagem do Rio Camaquã - Aspectos biológicos, físicos e químicos da água. Relatório técnico final, Vol. I - Terceira parte, São Leopoldo, Unisinos. 333p.

KONRAD, H. G. \& PALOSKI, N. I. 2000. Fauna da região das Minas do Camaquã, Sub-Bacia do Arroio João Dias. In Minas do Camaquã, um Estudo Multidisciplinar (L. H. Ronchi, \& A. O. C. Lobato, eds.). Unisinos, São Leopoldo, p.85-108.

KRÜGER, C. P. \& SILVA, E. J. E. 2003. Papilionoidea (Lepidoptera) de Pelotas e seus arredores, Rio Grande do Sul, Brasil. Entomologia y Vectores, 10(1):31-45.

LAMAS, G. 2004. Checklist: Part 4A, Hesperioidea - Papilionoidea, 479p. In: Atlas of Neotropical Lepidoptera (J. Heppner, ed.). Association for Tropical Lepidoptera, Scientific Publishers.
MARCHIORI. M.O. \& ROMANOWSKI, H.P., 2006. Borboletas (Lepidoptera: Papilionoidea e Hesperioidea) do Parque Estadual do Espinilho e entorno, Rio Grande do Sul, Brasil. Revta bras. Zool 23(4):1029-1037.

MINISTÉRIO DO MEIO AMBIENTE. 2000. Avaliação e ações prioritárias para a conservação da biodiversidade da mata atlântica e campos sulinos. Brasília, MMA-SBF, 40p.

MOTA, F. S. 1951. Estudos do clima do estado do Rio Grande do Sul segundo o sistema de W. Koppen. Revista Brasileira de Geografia, 13:275-284.

MOTTA, P. C. 2002. Butterflies from the Uberlândia region, central Brasil: species list and biological comments. Braziliam Journal of Biology, 62(1):151-163.

NEW, T.R.; PYLE, R.M.; THOMAS, J.A. \& HAMMOND, P.C. 1995. Butterfly conservation management. Annual Review of Entomology, 40:57-83.

OWEN, D.F. 1971. Tropical Butterflies. Carendon Press, Oxford, $\mathrm{XIV}+214 \mathrm{p}$

PILLAR, V.P., BOLDRINI, I.I., HASENACK, H., JACQUES, A.V.A, BOTH, R. 2006. Estado atual e desafios para a conservação dos campos. Instituto de Biociências, Universidade Federal do Rio Grande do Sul. http://ecoqua.ecologia.ufrgs.br/arquivos/Reprints\&Manuscripts/ Estado_Atual_e_Desafios_Conservacao_Campos_Workshop.pdf\#searc $\mathrm{h}=\% 22$ conserva\%C3\%A7\%C3\%A3o\%20dos $\% 20$ campos $\% 22$ (último acesso em 07/06/2006).

POLLARD, E. 1977. A method for assessing changes in the abundance of butterflies. Biological Conservation, 12:115-134.

PORTO, M.L. 2002. Os Campos Sulinos, sustentabilidade e manejo. Ciência \& Ambiente. 24:119-138.

QUADROS, F. L. F. \& PILLAR, V. P. 2002. Transições floresta-campo no Rio Grande do Sul. Ciência \& Ambiente, 24:109-118.

ROGO, L. \& ODULAJA, A. 2001. Butterfly populations in two forest fragments at the Kenya coast. African Journal of Ecology, 39:266-275

ROMANOWSKI, H. P. \& BUSS, G. 1997. Biodiversidade: animais brasileiros em extinção. In: Queridos animais: relações humanas e animais: novas áreas profissionais sob enfoque ecológico (A. Escoterguy, ed.). LP\&M, Porto Alegre, p.61-85.

SCHWARTZ, G. \& DI-MARE, R.A. 2001. Diversidade de quinze espécies de borboletas (Lepidoptera, Papilionidae) em sete comunidades de Santa Maria, RS. Ciênc. Rural, 31(1):49-55.

SHAHABUDDIN, G. \& TERBORGH, J.W. 1999. Frugivorous butterflies in Venezuelan forest fragments: abundance, diversity and the effects of isolation. Journal of Tropical Ecology, 15:703-722.

SPARROW, H.R., SISK, T.D., EHRLICH, P.R. \& MURPHY, D.D. 1993. Techniques and guidelines for monitoring neotropical butterflies. Conservation Biology, 8(3):800-809.

SUMMERVILLE, K.S., METZLER, E.H. \& CRIST, T.O. 2001. Diversity of Lepidoptera in Ohio forests at local and regional scales: how heterogeneous is the fauna? Annals of the Entomological Society of America, 94(4):583-591.

TYLER, H.A., BROWN, K.S. \& WILSON, K.H. 1994. Swallowtail butterflies of the Americas: A study in biological dynamics, ecological diversity, biosystematics and conservation. Scientific Publishers, Gainesville, 376p.

VIEJO J. L., DEVIEDMA M. G., FALERO E. M. 1989. The importance of woodlands in the conservation of butterflies (Lep, Papilionoidea and Hesperioidea) in the center of the Iberian Peninsula. Biological Conservation, 48(2):101-114.

Recebido em 29/08/06

Versão reformulada recebida em 15/11/07

Publicado em 28/01/08 